\title{
Immunoglobulin G4-related laryngeal disease
}

\section{A case series and literature review}

Benjamin Miller ${ }^{1}$, Chadwan Al-Yaghchi ${ }^{1}$, Justin Weir ${ }^{2}$, Alan Salama ${ }^{3}$, Khalid Ghufoor ${ }^{4}$, Guri Sandhu ${ }^{1}$

1. Department of Otolaryngology, Imperial College Healthcare NHS Trust

2. Department of Pathology, Imperial College Healthcare NHS Trust

3. Department of Nephrology, Royal Free London NHS Foundation Trust

4. Department of Otolaryngology, Barts Health NHS Trust

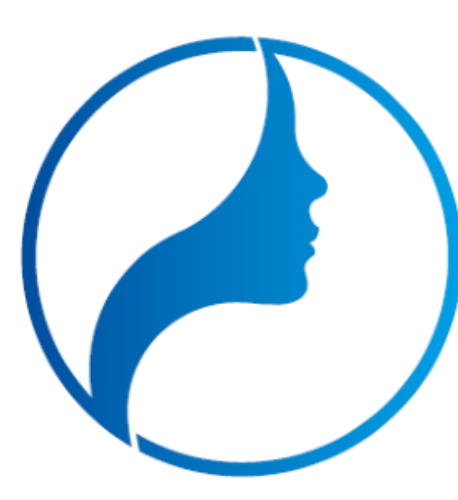

ENT • IMPERIAL

Correspondence: benjaminmiller@nhs.net

\section{Introduction}

Much progress has been made in our understanding of
Immunoglobulin G4-related disease (IgG4-RD), a chronic
inflammatory disorder that unites a variety of conditions previously
thought to be unrelated ${ }^{1-4}$. We describe three cases of laryngeal
IgG4-RD and a literature review of the subject.

\section{Case 1}

A 52 year old female presented to her local ENT department with dyspnoea and biphasic stridor. Flexible nasendoscopy (FNE) revealed marked supraglottic stenosis. Over 3 years she underwent repeat microlaryngobronchoscopy (MLB) dilatations and laser excision of interarytenoid fibrosis providing transient relief. Histology demonstrated inflammation with fibrosis, plasma cell infiltrates, and increased IgG4 plasma cells (Figure 1), and she was commenced on

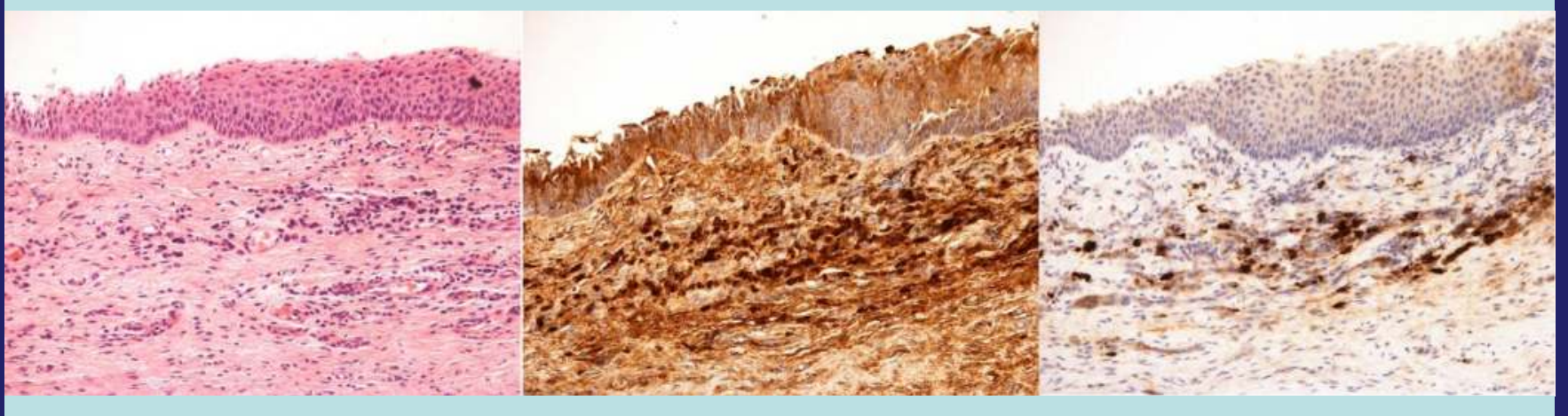

Figure 1. Histology demonstrating fibrosis with plasma cell infiltrates, IgG, $\operatorname{lgG4}$

oral prednisolone and azathioprine, later switched to methotrexate. Following a further relapse, she was referred to our institution where she underwent surgical tracheostomy and endoscopic laryngotracheal reconstruction (LTR), involving excision of interarytenoid scarring and insertion of a laryngeal stent covered with a superficial skin graft (Figure 2). The stent was removed at 1 week and she has been decannulated. She remains well with a good voice.

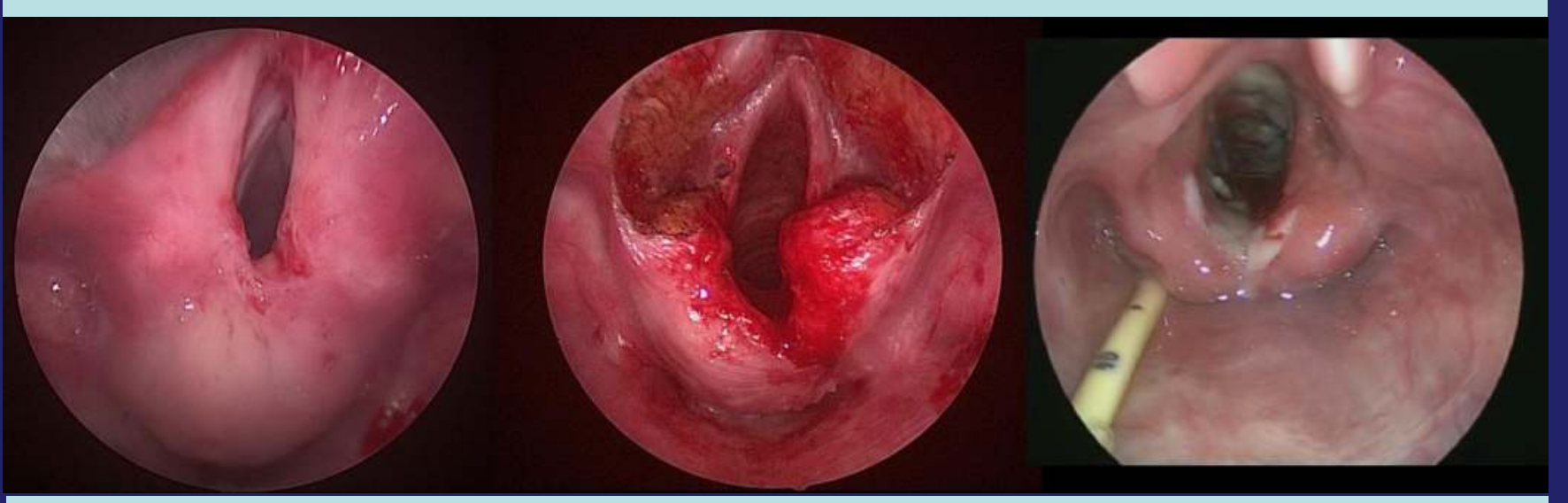

Figure 2. Supraglottic stenosis pre LTR, during LTR and on removal of stent

\section{Case $2^{5}$}

A 22 year old female presented to our institution with a 3 year history of idiopathic subglottic stenosis for which she had previously undergone tracheostomy. Histology reported inflammation only. On review FNE demonstrated complete subglottic occlusion. She underwent MLB and biopsy, revealing an inflammatory mass of lymphocytes and collagenised stroma with plasma cells. CT scan demonstrated abnormal soft tissue from the supraglottis to the tracheotomy. She was reviewed by a renal physician with expertise in autoimmune disorders (co-author AS) and commenced on prednisolone. Two months later she underwent LTR (Figure 3). Abnormal tissue was excised, the airway lumen obliterated, costal cartilage implanted for cricoid augmentation and a closed tracheal stent with skin graft inserted. The stent was removed at two weeks, and she was decannulated. She remains well with a normal voice. Histology demonstrated an IgG4 sclerosing pseudo-tumor.

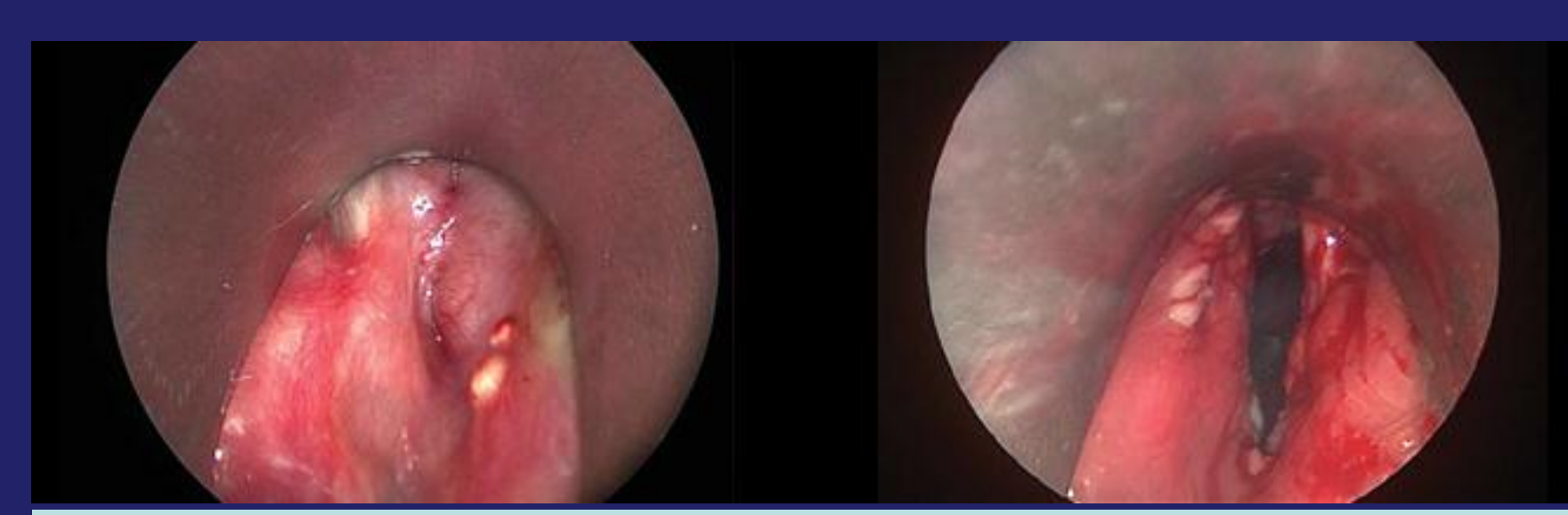

Figure 3. Intraoperative images of larynx before and after LTR

\section{Case 3}

A 15 year old female presented via A\&E with dyspnoea and biphasic stridor. FNE revealed subglottic stenosis. A CT scan confirmed subglottic narrowing to $4 \mathrm{~mm}$, and she underwent emergency laryngotracheoplasty. Restenosis required repeated MLB dilatations for transient relief, and a decision was made to perform a tracheostomy followed by LTR, using rib graft to the posterior cricoid and anterior augmentation with a split skin graft and sternohyoid flap. A subsequent CT demonstrated disease extension involving the right hemilarynx and supraglottis. Histology demonstrated an inflammatory sclerotic lesion with polyclonality on immunostaining, and she was referred to AS, who commenced her on azathioprine, and two courses of rituximab. Surveillance has demonstrated stable airway appearances, with a partial return of right vocal cord mobility, and she was decannulated the following year. Over the last 5 years peristomal tracheal scarring has warranted laser resection and the insertion of an indwelling tracheal stent with adjuvant sirolimus therapy. She remains well with a good voice, receiving intermittent stent changes (Figure 4).

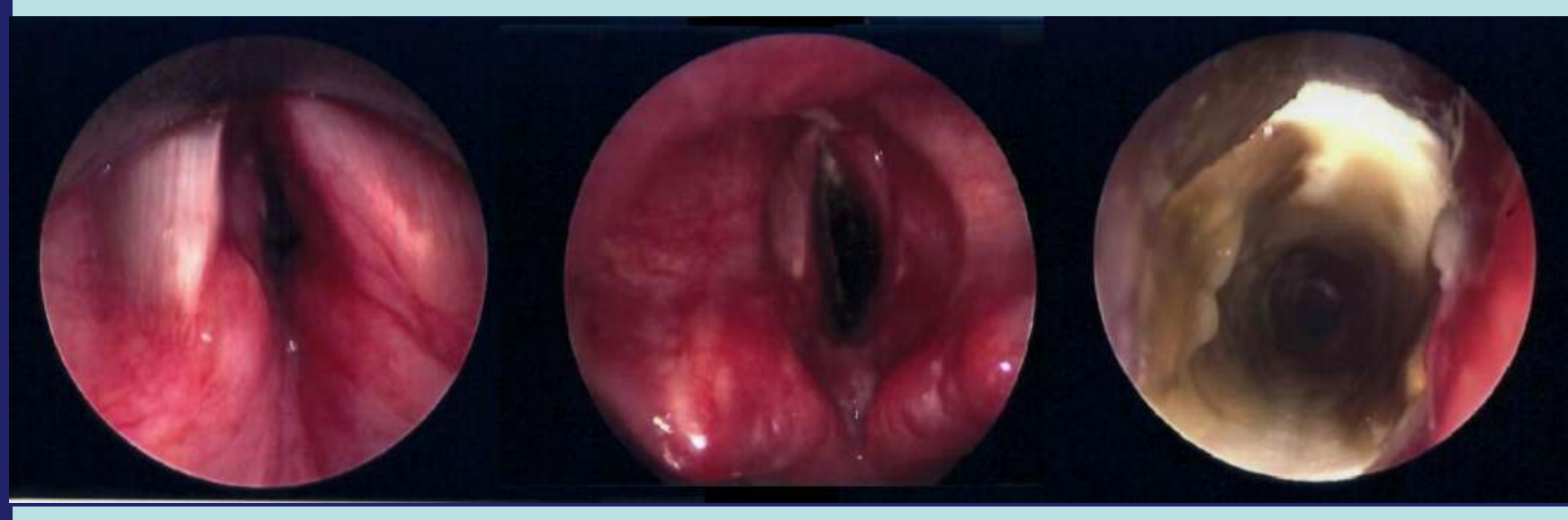

Figure 4. Subglottic stenosis pre LTR, post LTR, and at routine stent change 5 years on

\section{Literature Review}

A comprehensive review of the literature regarding laryngeal manifestations of IgG-RD was conducted. A total of 11 cases of laryngeal IgG4-RD have been reported and are summarised in a supplementary weblink below ${ }^{6-10}$. Seven cases required surgical resection, five required surgical tracheostomies, and four were treated successfully with immunotherapy alone.

\section{Conclusions}

In the 3 cases of laryngeal IgG4-RD described tissue biopsy was critical to diagnosis. Definitive management involved steroid and immunotherapy, and an individualized surgical approach based on site and extent of disease. All patients required tracheostomies but were decannulated, and have achieved good functional outcomes.

\section{References}

References and supplementary literature review summary available at https://tinyurl.com/BACOIgG4RD 